\title{
An investigation on the status of resilience among blind adolescent students
}

\author{
Tsigie Genet Zegeye
}

Bahir Dar University, Ethiopia

\begin{tabular}{|c|c|}
\hline Article Info & Abstract \\
\hline $\begin{array}{l}\text { Article History } \\
\text { Submitted: } 26 \text { August } 2018 \\
\text { Revised: } 2 \text { February } 2019 \\
\text { Published online: } 1 \text { April } 2019\end{array}$ & $\begin{array}{l}\text { Ethiopia has one of the world's highest rates of visual disability. Among } \\
\text { persons with visual disability living in Bahir Dar town, adolescents take a } \\
\text { significant share of this population. Living sightless for anyone, whether young } \\
\text { or old, educated or uneducated, rich or poor, with adequate support or without } \\
\text { it is seriously challenging. The quality of life of blind persons is significantly } \\
\text { influenced by the resilience they possess. The status of resilience of blind } \\
\text { adolescents learning in schools of Bahir Dar town is not known. Against this }\end{array}$ \\
\hline $\begin{array}{l}\text { Keywords } \\
\text { Bahir Dar } \\
\text { Blind adolescents } \\
\text { Resilience } \\
\text { Visually impairment }\end{array}$ & $\begin{array}{l}\text { resilience status of blind adolescent students and the influence of some } \\
\text { demographics on resilience. Data were collected from a random sample of } 60 \\
\text { blind adolescents using Connor-Davidson Resilience Scale. Descriptive } \\
\text { statistics, t-test, one-way ANOVA followed by Scheffe post hoc comparisons } \\
\text { were used to analyze the data. The results revealed lower level of resilience. } \\
\text { Gender, time of onset of blindness, parental education and family income were } \\
\text { influencing resilience. Implications for education and rehabilitation of blind } \\
\text { adolescents are explored. }\end{array}$ \\
\hline
\end{tabular}

\section{Introduction}

Absence of usable vision or the status of being blind for anyone disregarding his/her developmental period is badly limiting. This would be more so for blind adolescent since in addition to the already existing challenges and demands posed by blindness, adolescence as a developmental period throws up very many developmental demands and tasks to be successfully completed. In this turbulent developmental period adolescents are put through rapid cognitive, psychosocial and physical growth and development (Donnon \& Hammond, 2007; Larson \& Wilson, 2004) demanding extra effort to constantly pursue for resilient and successful life. The same scholars further observe that to achieve this, all adolescents, with or without blindness, are expected to successfully perform the various developmental tasks of this period. This becomes more challenging for blind adolescent as they are to handle the challenges of blindness and the developmental stage of adolescence parallel. How do blind adolescent students salvage their quality of life during their adolescence period, especially in the context of Ethiopia where natural and rehabilitative provisions and support for blind persons are to their least minimum? How

\footnotetext{
Address of Corresponding Author

Tsigie Genet Zegeye, Bahir Dar University, College of Education and Behavioral Sciences, Department of Special Needs and Inclusive Education, Director of Support and Service for Students with Disabilities, P O Box 79, Bahir Dar, Ethiopia.

\tsigie1968@yahoo.com

0000-0002-5990-2039

How to cite: Zegeye, T. G. (2019). An investigation on the status of resilience among blind adolescent students. Journal of Pedagogical Research, 3(1), 50-59. doi: 10.33902/JPR.2019.4
} 
resilient are blind adolescent students in Ethiopian context is a pertinent question need answers to meaningfully and effectively support this vulnerable population in this Country.

The consequences of losing the sense of sight either partially or totally, before birth or later in life can lead to significant negative impacts on an individual's short- and long-term welfare and development (Clarke \& McKay, 2008; Glenda, Elaine \& Anita, 2010; Lowenfield, 1981; Ürey \& Güler, 2018). Loss of vision affects all areas of development of individuals and seriously limits their ability to learn incidentally from their environments (Lowenfield, 1981). In Ethiopia, persons with disabilities in general and blind individuals in particular are viewed as not capable of supporting themselves or establishing rewarding relationships with others and hence cannot be resilient (Berhane, Alemayehu, \& Abebe, 2006; Tirussew, 2005). Amidst such unfounded beliefs, some blind adolescents succeed in their education despite exposure to various barriers and challenges at different levels of their environment (Tirussew, 2005). Nevertheless, the case with most of the blind adolescent is not the same; they live a mere sub-human life. Adolescents who succeed in spite of adversity have been identified as resilient (Zolkoski \& Bullock, 2012).

Resilience was described as a trait that moderates the negative effects of barriers and adversities, and promotes adaptations (Wagnild \& Young, 1993). While some people seem born with resilience characteristics, many of these resilience characteristics can be developed and strengthened, which can increase one's ability to deal with life's challenges (Levine, 2003). Resilient people tend to be skilled in preparing for adversities and competent at accepting what comes at them with flexibility rather than rigidity. They are like bamboo in a hurricane, they bend rather than break or even if they feel like they are broken for a time, there is still a part of them deep inside that knows they will not be broken permanently (Dahlbeck \& Lightsey, 2008; Waters, 2013). Hence, knowing the levels of resilience of people living under a specific context has paramount significance.

Research into resilience of the typical population has progressively increased over the last five decades. Indeed, when it comes to the experience of disability, resilience is implied as an attribute of the individual with disability (Ellis, 2003). As a result, adolescents with disabilities are all too often excluded from the category of resilient persons simply because they have impairments and (Runswick-Cole \& Goodley, 2013) and are relegated to the category of the vulnerable and the passive (Goldstein \& Brooks, 2013).

Resilience studies illuminating the perspectives of persons with disabilities in general and blind adolescent in particular are seriously lacking (Hart et al., 2013). Luthar and Zelazo (2003) rightly indicated that one of the weaknesses of research on resilience has been the exclusive focus on children and adolescents without disability. Blind adolescent attending schools, locked up at homes, struggling with navigation on roads, begging on streets and places of worships, etc. capture the lives of blind persons living in Bahir Dar town, the capital city of Amhara Regional State. Barring a very few privileged ones, the lives of the brutal majority of blind adolescent here are deplorable implying that it is very close to non-human existence. However, no clear understanding exists as to why some in this population are resilient while the majority are not as resilience of blind adolescents had never captured the attention of disability related researchers of Ethiopia. In the context of Ethiopia we still do not know the level of resiliency of blind adolescents.

\subsection{Rationale and Objectives}

The legal climate existing at present in Ethiopia grant persons with disabilities the right to appropriate and relevant support (EFDRE, 1987; MoE, 1994), including blind adolescent students. However, the available evidence suggests that the situation of blind adolescents is neither exciting nor encouraging. Nevertheless, some blind adolescents emerge resilient and successful in life. How? A definite answer eludes as no systematic studies attempted to find an answer to this broad and vital question. As a result, the level of resilience of blind adolescents living in Ethiopia is still unknown. Against this backdrop, this study was germinated to assess the level of resilience of blind adolescents living in Bahir Dar town. The study also aimed at investigating the way the type 
of onset of blindness and some selected demographic variables relate to resilience among blind adolescents. It is hoped that an understanding of the crucial roles that resilience plays in the lives of blind adolescent would invariably inform all the education and rehabilitation efforts for blind adolescents.

\section{Method}

\subsection{Design of the Study}

A survey design was employed to examine the resilience status of blind adolescent students attending their education in schools of Bahir Dar town. This design is appropriate when investigating specific variables of a proposed study and when seeking to discover possible relationships between groups of independent and dependent variables (Brink \& Wood, 1998). Thus, using this deign the resilience status of blind adolescents were investigated.

\subsection{Sample}

Blind adolescent students between the ages of 13 and 19 years who were studying at six primary schools, three secondary schools and three preparatory schools of Bahir Dar town constituted the population of the study. While primary schools are medium sized, secondary and preparatory schools are large. The environment of these schools is currently not suitable for blind students. Of the total of 200 (108 males and 92 females) blind adolescent student population of these schools, 60 (32 males and 28 females) were drawn as sample following stratified proportionate random sampling technique.

\subsection{Instrument}

Connor-Davidson Resilience Scale (CD-RISC) was the instruments used in this study. CD-RISC is a psychometrically strong 25 item scale rated on a five point Likert scale with response alternatives ranging from 0 (not true at all) to 4 (true nearly all the time). Scores are summed up to determine the total resilience score which can range from 0 to 100 . Higher scores reflect greater resilience. CD-RISC was reported to have sound psychometric properties with greater reliability and validity compared to other resilience scales (Goins, Gregg, \& Fiske, 2013). It was tested across different groups of respondents for reliability and the results yielded an average Cronbach's alpha of 0.89 and item total correlations ranged from 0.30 to 0.70 . A review of studies which used the CD-RISC demonstrated that CD-RISC is a valid instrument for measuring individual's resilience in a variety of populations such as large community samples, survivors of various traumas, caregivers of persons with Alzheimer's disease, adolescents, elders, patients in treatment for PTSD, members of different ethnic groups and cultures and selected professionals or athletic groups (see Connor \& Davidson, 2003). The English version of CD-RISC was translated to Amharic (the mother tongue of the participants and the national language of Ethiopia) following all the rigors of instrument translation and the Amharic version was used for data collection. An internal consistency reliability check was conducted using the final data collected for the study and the Cronbach's alpha was found to be 0.92 .

\subsection{Procedures of Data Collection}

On completion of pre-data collection preparations, four data collectors were recruited and trained on data collection procedures. The training included contents on rapport creation, respecting the respondent, reading the items without exerting an influence on response selection by the participants, entry of data in the instrument pack, etc. The survey was administered in a paper and pencil format with the participant sitting near to the data collector in private settings. Since the participants were unable to read print materials, the data collectors read everything contained in the instrument and made sure that respondents understood everything which is read. Participants were informed of the purpose of the survey and the ethical guarantees in place. Subsequently the data collector read item by item, secured the responses from the respondents and entered into the 
instrument. The participants were provided with the opportunity to ask any clarification at any point in time during data collection.

\subsection{Data Analysis}

The data were entered into the SPSS software and checked and edited in preparation for analysis. Descriptive statistics were used to calculate the levels of resilience. Independent samples t-test, ANOVA and Scheffe post hoc pair wise comparisons were employed to compare the resilience of participants grouped based on some demographics.

\section{Results}

\subsection{Levels of Resilience}

On a possible score range of zero to 100, the sample scored a mean resilience score of 40.60 $(\mathrm{SD}=9.28)$. The scores ranged from 18 to 76 . Considering a score falling towards the maximum possible score as stronger resilience and scores closer to the minimum possible score as weak resilience, the mean score of the sample can be interpreted as significantly lower to 100 and also strikingly lower than the mid value of the scale, i.e., 50 .

\subsection{The Relationship between Demographics and Resilience}

Sub-samples were formed based on the sample's gender, onset of blindness, education of father and mother and family income. Independent samples t-test, one-way ANOVA and post hoc pair wise comparisons were employed to examine if significant differences existed as a function of the demographics mentioned above. Independent samples $t$ - test between males $(M=44.36$, $\mathrm{SD}=10.31)$ and females $(\mathrm{M}=35.19, \mathrm{SD}=9.56)$ yielded a statistically significant mean difference $\left(t_{(58)}=2.29, p<.05\right)$. In order to examine if onset of blindness influenced resilience, participants were grouped into adventitiously $(M=43.26$, $S D=12.80)$ and congenitally $(M=36.54, S D=11.82)$ blind. Independent samples t-test revealed significant mean difference between adventitiously and congenitally blind adolescents students $\left(t_{(58)}=2.16, p<.05\right)$.

To explore if level of education of parents influenced resilience, mothers' and fathers' educational status were considered separately. This was done because it was assumed that mothers exert a greater role in the lives of blind adolescent students in the context of Ethiopia and hence assessing the association of parental educational status on resilience separately would be more informing. To this end, four groups of adolescents were formed based on their mothers' educational status; viz., non-educated $(\mathrm{M}=30.89$, $\mathrm{SD}=6.46)$, primary education $(\mathrm{M}=31.70$, $\mathrm{SD}=9.13)$, secondary education $(\mathrm{M}=46.37, \mathrm{SD}=9.38)$ and tertiary education $(\mathrm{M}=54.98$, $\mathrm{SD}=9.42)$. Result of one way ANOVA indicated a significant mean difference among the groups compared $\left(F_{(3,56)}=17.51, p<.001\right)$. Further, Scheffe post hoc comparisons revealed significant mean differences in four out of the six comparisons made revealing a general trend that as level of education of mothers increases, the resilience of their blind adolescents increases. That is, blind adolescent students whose mothers were tertiary educated had significantly higher resilience than blind adolescent students whose mothers were non-educated (MD $=24.07, p<.001$ ) and primary educated (MD $=23.26, p<.001)$. Further, adolescent children of secondary educated mothers had significantly higher resilience in comparison with blind adolescent students whose mothers were primary educated $(\mathrm{MD}=14.67, p<.001)$ and non-educated $(\mathrm{MD}=15.48, p<.001)$. However, children of non-educated mothers did not significantly differ on their resilience from adolescents with mothers having primary education. Further, no significant difference emerged between adolescents whose mothers had secondary and tertiary education.

Similarly, adolescents were categorized into the same four groups based on their fathers' educational status, namely, non-educated $(M=28.35, S D=7.13)$, primary educated $(M=34.40$, $\mathrm{SD}=6.30)$, secondary educated $(\mathrm{M}=42.72, \mathrm{SD}=9.54)$, and tertiary educated $(\mathrm{M}=55.84$, $\mathrm{SD}=10.05)$. One way ANOVA results revealed a statistically significant mean difference among the groups on their resilience $\left(F_{(3,56)}=19.512, p<.001\right)$. Scheffe post hoc test resulted in significant 
mean differences in four out of six pairs of comparisons made. As in the case of mothers' education, a general trend that as fathers' level of education increases, resilience of their adolescent children increases emerged. That is, blind adolescent students whose fathers were tertiary educated had significantly higher resilience than blind adolescent whose fathers were secondary educated $(\mathrm{MD}=13.19, p<.001)$; primary educated $(\mathrm{MD}=21.44, p<.001)$; and non-educated $(\mathrm{MD}=27.49, p<.001)$.Further, Adolescent children of secondary educated fathers had significantly higher resilience in comparison with blind adolescent whose fathers were non-educated $(\mathrm{MD}=14.37, p<.001)$. However, children of non-educated and primary educated fathers and primary educated and secondary educated fathers did not differ significantly on their resilience.

The quality of life of anyone is greatly influenced by one's income. To examine if the family income influenced resilience of blind adolescent, the sample was grouped into five groups based on their total family income in Ethiopian Birr (1 $\$=$ approximately 29 Birr). The sub-samples thus formed were with the monthly income from 1000-2000 $(\mathrm{M}=28.43$, SD = 6.53), 2001-3000 $(\mathrm{M}=32.22, \mathrm{SD}=4.80), 3001-4000(\mathrm{M}=40.40, \mathrm{SD}=8.14), 4001-6000(\mathrm{M}=49.82, \mathrm{SD}=9.79)$ and greater than $6001(\mathrm{M}=56.60, \mathrm{SD}=10.64)$ Birr. Result of one way ANOVA yielded statistically significant mean difference on resilience $\left(\mathrm{F}_{(4,55)}=16.54, p<.001\right)$. Follow-up Scheffe post hoc pair wise comparison indicated a pattern of difference wherein blind adolescents from lower income families having significantly lower level of resilience than adolescents from highest income groups. The detailed results are shown in Table 1.

Table 1.

Results of Scheffe post hoc comparisons across level of family income

\begin{tabular}{lllll}
\hline \multicolumn{5}{c}{ Level of income } \\
\hline Level of income & $2001-3000$ & $3001-4000$ & $4001-6000$ & $>6000$ \\
$1000-2000$ & -3.794 & -11.971 & $-21.395^{* *}$ & $-28.071^{* *}$ \\
$2001-3000$ & & -8.178 & $-17.601^{* *}$ & $-24.278^{* *}$ \\
$3001-4000$ & & & -9.424 & $-16.100^{* *}$ \\
$4001-6000$ & & & & -6.676 \\
\hline
\end{tabular}

$* * p<.01$

\section{Discussion}

\subsection{Levels of Resilience of Blind Adolescent Students}

The mean resilience score of 40.60 on a possible score range of zero to 100 invariably implies weak resiliency and hence an undesirable position for any population. Though 100 could be considered ideal and unrealistic, especially for persons living with blindness in third world countries, a mean score closer to 100 or significantly above 50 -the middle value of the possible score range, would have been an encouraging result. Indicating a weak status of resilience, the sample's mean score fell below the median scale value. Blind adolescent would require stronger and higher level of resilience than adolescents without visual loss as the demands placed on blind adolescent by their sensory limitations as well as environmental barriers would demand extra capacities to tackle the day-to-day demands. This becomes more so for blind adolescent $\mathrm{s}$ who live in underdeveloped or developing world since habilitation and/or rehabilitation service, inclusive provisions and infrastructure in environments are very much limited, if not non-existent, in such societies.

\subsection{Resilience and Demographics}

Resilience development may vary based on the social and environmental resources available for a person. These resources may not be equally provided or available for males and females across different cultures. In a culture were disability is misperceived and stigmatized, persons with disabilities face lots of deprivations and maltreatments at different levels of their environment. This maltreatment and neglect may be more severe when it comes to females with a disability 
especially in less developed nations such as Ethiopia (Tirusew, 2005) as gender inequality is the order of the day even today in such developing nations. As expected, the results of the present study indicated a statistically significant resilience mean difference between males and females wherein males have greater resilience than females. Findings of previous studies coming from other contexts on the association between resilience and gender were inconsistent. While some studies indicated the absence of associations between resilience and gender (e.g., Wagnild \& Young, 1997), several other studies found strong associations where the level of resilience was higher in females than males (e.g., Sun \& Stewart, 2012). These studies attributed greater resilience of females to the presence of more positive connections of females with parents, teachers, adults in the community and peer relations and autonomy experiences than males. In contrast, going along with the current result, Friburg, Barlang, Martinussen, and Rosenvinge (2005) and Bonanno (2004) reported male gender predicting increased likelihood of resilient outcomes than female gender. Further, Bonanno, Galea, Bucciarelli and Vlahov (2007) observed women as less than half as likely to be resilient as men. All these inconsistent results on the association between resilience and gender appear to inform that resilience development varies across contexts and cultures, be it in males or females and that is depended upon the availability of protective resources in a specific context at a given point in time. In Ethiopia, though women are respected and protected, they are placed far below than men in their social significance. Women here traditionally have been considered as child bearers, home makers and less to contribute to the economic resources of the family and society. Hence for women in general and women with visual disabilities in particular, protective resources at different levels of the environment may not be made available as it is provided for men. Blind women are at double disadvantage for being women and having visual disability. The lower social status assigned to women coupled with the stigma and stereotypes attached to disability may be jointly contributing to their lower level of resilience in comparison with men. Since this study is not in a position to make such a conclusion, data based, further researches are signaled.

Another demographic that was hypothesized to influence resilience was the time of onset of blindness. Blindness that happens at birth or shortly afterwards (congenital) and acquired later in life (adventitious) will not have the same impact on resilience development as the psychological and day-to-day demands and challenges generated by the time of onset are drastically different. With this presumption, when adventitiously blind respondents were compared with congenitally blind respondents, a statistically significant difference in resilience emerged wherein persons with adventitious blindness were found to be more resilient than those with congenital blindness. On the association between time of onset of blindness and resilience, the existing literature is very much inconsistent. For instance, Bonanno (2004) explored and compared the level of resilience among the sighted, congenitally and adventitiously blinded persons. The results revealed that persons with congenital blindness had higher levels of resilience among all the three groups. Another study, consistent with the result of the present study however, revealed that persons with adventitious blindness had greater resilience than those with congenital blindness (Zeeshan \& Aslam, 2013). This may be because individuals with adventitious blindness may retain significant visual memory to profit from descriptions of a visual nature. Even when they retain no visual memory, they still hold the advantage of their previous visual learning, which would motivate them to move about, discover and interact with their environment. They are often more active, curious and better coordinated than persons with congenital blindness (Bonanno, 2004). Further, the secondary and tertiary preventive measures in place in the environment where persons having blindness live, though vital for both congenitally and adventitiously blind individuals are of paramount importance for congenitally blind persons. The reason being, they are to capitalize on to such services available for their day-to-day life as they don't have or retain any visual memory. Provisions aiming to rehabilitate or habilitate blind adolescent in Ethiopia are strikingly inadequate as well as inefficient. Stated otherwise, the protective resources available at various environmental in Bahir Dar town may be very much inadequate for congenitally blind persons 
than adventitiously blind. Persons with blindness living in such societies are expected to face serious challenges, impeding their resilience. This is all the more true for congenitally blind persons. Such an insight looks more grounded in the context in which this study was conducted.

The role of education in minimizing the negative attitudes of parents towards their children with a disability and to a positive outcome of parenting is unassailable across contexts. With this view in mind, the association between mothers' and fathers' education and the resilience of their adolescent offspring was examined separately. The results showed a clear association between mothers' and fathers' education and the resilience of their adolescent children. In both the cases a near perfect trend exists, that is, when the level of education of fathers and mothers increases the level of resilience of their adolescent children also increases. What are the ways with which parental education influence the resilience of children? Since this study did not try to answer this question, future researches in those lines look imperative. However, the existing research evidences suggest that parents with better education provide appropriate support and expect their children with disabilities to obtain better grades and want them to proceed the highest level of education that they can whereas less and uneducated parents provide lesser support and have lower academic expectations for their children with disability (Benner \& Mistry, 2007; McLeod \& Owens, 2004; Ungar, 2004; Wathum-Ocama \& Rose, 2002). What changes in parental attitudes towards disability in general and blindness in particular, provision of disability specific support to their children having blindness, parental involvement in the education of children with blindness, and parental expectations about the academic achievement and progression would be brought about by parental education are pertinent questions which need to be answered so as to capitalize on parental education in resilience development of blind adolescent students. Such insights would invariably inform and aid all the habilitation and/or rehabilitation efforts aimed at enhancing the quality of life of blind adolescent students.

Family income can well predict the quality of life of its members, that is, the higher the income the better the quality of life. Assuming that blind adolescent students living in higher income families would be exposed to lesser risks and more resources at family level and also would be able to purchase better services outside, the association between family income and the level of resilience of blind adolescent students was pondered into. As hypothesized, blind adolescent students from higher income families do possess higher level of resilience in comparison with their counterparts from lower income families. A closer scrutiny reveals that blind adolescent students with a family income of less than 4000 Birr are less resilient than those with more than the family income of 4000 Birr. Even though a clear trend of increased resilience in accordance with the categorization of family income groups made for this study does not emerge, the general pattern of results clearly indicates the association between income and resilience among blind adolescent students.

How family income does come around and influence resilience of its members with blindness? Data based answers to this question are out of the purview of this study though they are of absolute importance. It looks logical to infer that when parents come short of enough finance, the needs of a blind child may remain unmet and it may also affect their care giving behavior. Whereas parents who have better monthly income may be able to provide better support for their blind adolescent students. As a result, higher income as well as affluent families would be able to purchase higher quality education and other habilitation and rehabilitation services for the blind adolescent students. This in turn can nurture their resilience better. This result aligns well with the findings coming from other contexts where positive associations between income and resilience was found in a pattern in which lower income to be associated with lower level of resilience (e.g., Benner \& Mistry, 2007; Wagnlid, 2003) and higher income to be associated with higher levels of resilience (e.g., Hardy et al., 2004; McLeod \& Owens, 2004; Wathum-Ocama \& Rose, 2002). 


\section{Conclusions and Implications}

This study brings out a comprehensive picture of the status of blind adolescent students resilience under the present context of Bahir Dar, Ethiopia. Blind adolescents currently living in Bahir Dar are less resilient. Gender has a significant influence on resilience; male blind adolescent students are more resilient than female adolescents. Adolescents who were adventitiously blind are more resilient than those congenitally blind, indicative of the influence played by the time of onset of blindness in resilience development. Mothers' as well as fathers' education positively contribute to the resilience of blind adolescent students. Family income too influences resilience of blind adolescent students in a manner in which higher family income goes with higher resilience and the vice versa.

The findings of this study obviously highlight the tremendous influence that environmental barriers and resources exert on the development of resilience among blind adolescent students. Though there may be certain resilience characteristics that an individual is born with that are instrumental to resilience development, these factors are differentiated by context and demographic characteristics of persons with blindness, and thus they are subject to change. This has strong implication for all the habilitation and rehabilitation efforts. Helping blind persons to live independently and productively as much as possible in society is the ultimate objective of any rehabilitation and habilitation efforts. The philosophy of quality of life underpins such efforts too. They can be achieved only if resilience of blind persons is enhanced. Here comes the contribution of this investigation. Since lack resources (e.g. lower levels of income and education of parents) impede resiliency, reducing or eliminating these challenges to the fullest possible extent would go a long way in strengthening resilience. The positive contribution of resources imply that the rehabilitation and habilitation efforts should place an extra thrust on to developing and/or introducing protective resources at different levels of the environment. The findings of the study also imply the roles that the schools in Ethiopia should play in building resilience of blind students. The result also suggest that inclusive education is not just about the placement of children with blindness into regular schools but about the delivery of educational services customized to their needs and the provision of adequate materials and human assistance to eliminate the barriers to inclusion and inclusive education. An integrated effort to reduce barriers and increase resources at home and school through the provision of disability specific support services so as to build the resilience capacity of blind adolescent students is the pertinent implication of this study. Blind students who live and operate in such home and school environment would naturally be more resilient which in turn would enhance the quality of their lives.

\section{References}

Berhane, Y., Alemayehu, W., \& Abebe, B. (2006). National survey on blindness, low vision and trachoma in Ethiopia. Retrieved

from http://www.pbunion.org/Countriessurveyresults/Ethiopia/Ethiopian_National_Blindness_and_tracho ma_survey.pdf.[12 May 2017].

Benner, A., D., \& Mistry, R., S. (2007). Congruence of mother and teacher educational expectations and lowincome youth's academic competence. Journal of Educational Psychology, 99, 140 - 53.

Bonanno, G., A. (2004). Loss, trauma, and human resilience: have we underestimated the human capacity to thrive after extremely aversive events? American Psychology, 59(1), 20- 28.

Bonanno, G. A., Galea, S., Bucciarelli, A., \& Vlahov, D. (2007). What predicts psychological resilience after disaster? The role of demographics, resources, and life stress. Journal of Consulting and Clinical Psychology, 75, 671- 682. doi:10.1037/0022 - 006X.75.5.671

Brink, P., J., \& Wood, M., J. (1998). Advanced design in nursing research. Thousand Oaks: Sage.

Clarke, H., \& McKay, S. (2008). Exploring disability, family formation and break-up: reviewing the evidence. Birmingham: Crown. 
Dahlbeck, D., \& Lightsey, O. (2008). Generalized self-efficacy, coping and self-esteem as predictors of psychological adjustment among children with disabilities or chronic illnesses. Children's Health Care, 37, 293-315.

Connor, K. M., \& Davidson, J. R. T. (2003). Development of a new resilience scale: the Connor-Davidson Resilience Scale (CD-RISC). Depression and Anxiety, 18(2), 76-82.

Donnon \& Hammond (2007). A psychometric assessment of the self-reported youth resiliency: Assessing developmental strengths questionnaire. Psychol Rep., 100, 963-78.

Ellis, K., M. (2003). Breakdown is built into it: a politics of resilience in a disabling world. Media and Culture Journal,16, $5 . \quad$ Retrieved from http://researchrepository.murdoch.edu.au/id/eprint/37281/1/Breakdown.pdf [12 December 2017].

FDRE (1987). Federal Democratic Republic of Ethiopia Constitution. Bahir Dar: EMPDA.

Friburg, O., Barlang, D., Martinussen, M., Rosenvinge, J. (2005). Resilience predicting psychiatric symptoms: a prospective study of protective resources and their role in adjustment to stressful life events. International Journal of Psychiatry, 14, 29-42.

Glenda, M., Elaine, C., \& Anita, B. (2010).The treasure in leisure activities: Fostering resilience in young persons who are blind. Journal of Visual Impairment, 104(7), 419-430.

Goins, R., T., Gregg, J., J. \& Fiske, A. (2013). Psychometric properties of the Connor-Davidson Resilience Scale with older American Indians: the native elder care study. Resilience Aging, 35(2), 123-143. doi: $10.1177 / 0164027511431989$.

Ürey, M. \& Güler, M. (2018). A qualitative study on how students with visual impairments perceive environmental issues. Journal of Science Education for Students with Disabilities, 21(1), 15-28.

Hardy, S., Concato, J., \& Gill, T. (2004). Resilience of community-dwelling older persons with blindness. Journal of the American Geriatrics society, 52(2), 257-262.

Hart, A., Heaver, B., Brunnberg, E., Sandberg, A., Macpherson, H., Coombe, S., \& Kourkoutas, E. (2013). Resilience-building with disabled children and young persons: a review and critique of the academic evidence base. International Journal of Child, Youth and Family Studies, 5(3), 394-422.

Katherine, R., Dan, G., \& Rebecca, L. (2014). Resilience in the lives of disabled persons across the Life Span. London: RoutledgeFalmer.

Larson, R., \& Wilson, S. (2004). Adolescence across place and time: Globalization and the changing pathways to adulthood. In R. Lerner \& L. Steinberg (Eds.). Handbook of adolescent psychology (229-330). New York: Wiley.

Levine, S. (2003). Psychological and social aspects of resilience: A synthesis of risk and resources. Dialogues Clinical Neuroscience, 5(3), 273-280.

Lowenfeld, B. (1981). Blindness and blind persons. New York: American Foundation for the Blind.

Luthar, S. S., \& Zelazo, L. B. (2003). Research on resilience: an integrative review. In S.S. Luthar, resilience and vulnerability: Adaptation in the context of childhood adversities. (pp. 510-549). New York: Cambridge University Press.

Major, B., Richards, C., Cooper, M. L., Cozzarelli, C., \& Zubek, J. (1998). Personal resilience, cognitive appraisals and coping: an integrative model of adjustment to abortion. Journal of Personality and Social Psychology, 74, 735-752.

McLeod, J. D., \& Owens, T. J. (2004). Psychological well-being in the early life course: Variations by socioeconomic status, gender, and race/ethnicity. Social Psychology Quarterly, 67(3), 257 - 278.

MoE (1994). Transitional government of Ethiopia education and training policy. Addis Ababa: EMPDA.

Runswick-Cole, K. \& Goodley, D. (2013). Resilience: a disability studies and community psychology approach, social and personality psychology. Compass, 7(2), 67-78.

Sun, J., \& Stewart, D. (2012).Age and gender effects on resilience in children and adolescents. International Journal of Mental Health Promotion, 9(4), 16-25.

Tirussew, T. (2005). Resilience and success of persons with disabilities in Ethiopia. Addis Ababa: Addis Ababa University Printing Press.

Wagnild, G. (2003). Resilience and successful aging: comparison among low and high income older adults. Journal of Gerontological Nursing, 29(12), 42- 49.

Wagnild, G., \& Young, H. (1997). Resilience among older women. Journal of Nursing Scholarship, 22(4), 252255.

Wagnild, G., M.,\& Young, H., M. (1993).Development and psychometric evaluation of the resilience scale. Journal of Nursing Measurement, 1(2), 165 - 178. 
Wathum-Ocama, J., C., \& Rose, S. (2002). Hmong immigrants' view on the education of their deaf and hard of hearing children. American Annals of the Deaf, 147(3), 44-53.

Zeeshan, M., Aslam, N. (2013). Resilience and psychological well- being among congenitally blind, late blind and sighted individuals. Journal of Educational Research and Studies, 1, 1-17.

Zolkoski , S., \& Bullock, M. (2012). Resilience in children and youth: a review. Children and Youth services, 34, 2295-2303. 